\title{
First observation of a transverse vertical oscillation during the formation of a hot post-flare loop
}

\author{
R. S. White, E. Verwichte, and C. Foullon \\ Centre for Fusion, Space and Astrophysics, Department of Physics, University of Warwick, Coventry CV4 7AL, UK \\ e-mail: r.s.white@warwick.ac.uk
}

Received 20 June 2012 / Accepted 24 July 2012

\begin{abstract}
Aims. We report and analyse the first observation of a transverse oscillation in a hot coronal loop with the Atmospheric Imaging Assembly (AIA) on the Solar Dynamics Observatory (SDO), following a linked coronal-flare mass-ejection event on the 3 November 2010. The oscillating coronal loop is observed off the east solar limb and exclusively in the $131 \AA$ and $94 \AA$ bandpasses, indicating a loop plasma of temperature in the range of 9-11 MK. Furthermore, the loop is not observed to cool into the other AIA channels, but just disappears from all bandpasses at the end of the oscillation.

Methods. A time series analysis of the loop oscillation is conducted by taking several cuts at different positions along the loop, estimating the transverse displacements over time for two strands in the loop and fitting those with a damped cosine curve. Intensity time variations, both along the loop and for a series of cut cross-sections, are investigated. Using a three-dimensional loop geometry obtained from a comparison of STEREO-B/EUVI and AIA images, we model different modes of transverse oscillations in the uniformly filled loop.

Results. Our time series analysis reveals a period of $302 \pm 14 \mathrm{~s}(291 \pm 9 \mathrm{~s})$ and a damping time of $306 \pm 43 \mathrm{~s}$ ( $487 \pm 125 \mathrm{~s})$ for the first (second) loop strand. A spatial phase shift along the loop of approximately $180^{\circ}$ suggests that we observe a higher order harmonic. Intensity oscillations are consistent with an interpretation in terms of a vertically polarised mode. Our forward modelling suggests that the loop oscillates as either a second or third order harmonic of this mode.

Conclusions. This is the first observation of a transverse loop oscillation observed exclusively in the hot coronal lines. The loop oscillation is vertically polarised and is dominated by a higher order harmonic mode. We conclude that the excitation mechanism of this 5 min period oscillation is directly connected with the reconnection processes that form the post flare loop, which differs from the blast wave excitation mechanism often proposed as the cause of cooler transverse loop oscillations.
\end{abstract}

Key words. magnetohydrodynamics (MHD) - Sun: corona - Sun: oscillations - Sun: UV radiation - Sun: flares

\section{Introduction}

Coronal oscillations in hot flare lines such as Fe XIX (6.3 MK) and Fe XX (8 MK) have been observed with the Solar Ultraviolet Measurements of Emitted Radiation (SUMER) spectrometer on SOHO (Kliem et al. 2002; Wang et al. 2002, 2003a,b). The SUMER line-of-sight Doppler-shift observations of 27 events originating from limb active regions performed by Wang et al. (2003b) revealed oscillations with periods between $7 \mathrm{~min}$ and $31 \mathrm{~min}$ and in a few cases intensity variations that lagged behind the Doppler shift velocities by a quarter period. Other observations utilising crystal spectrometers have also detected oscillations in flare regions. During the decay of a limb flare, Seely \& Feldman (1984) detected a damped oscillation with a period of about $10 \mathrm{~min}$ with the solar flare X-rays (SOLFLEX) crystal spectrometer (Ca XIX line) on the P78-1 satellite. Heavily damped oscillations with periods of $3.9 \mathrm{~min}$ and $3.6 \mathrm{~min}$ and damping times of 3.5 min and 4.3 min observed with the Bragg Crystal Spectrometer (BCS) on Yohkoh were also reported (Mariska 2005). Both the SUMER and BCS oscillations were interpreted as standing slow magneto-acoustic modes because (i) the estimate of the phase speed was close to the value of the sound speed; (ii) in a few cases associated intensity variations were found to lag the Doppler shift velocities by roughly a quarter period and (iii) average oscillation periods were found to be significantly longer than transverse oscillations observed by the Transition Region and Coronal Explorer (TRACE), suggesting that they constitute a different type of oscillation. We refer to Wang (2011) for a detailed review of standing slow modes in hot coronal loops.

The SUMER observations share some features with transverse oscillations, such as their impulsive excitation, short damping times, and associated intensity variations. The BCS oscillations have periods and damping times consistent with those for transverse loop oscillations (TLOs). Transverse oscillation periods longer than $10 \mathrm{~min}$ have been observed (Hori et al. 2005; De Moortel \& Brady 2007; Van Doorsselaere et al. 2009; Verwichte et al. 2009, 2010), which puts them in the range of those periods observed with SUMER. Tothova et al. (2011) detected in combined TRACE and SUMER observations the copresence of slow and transverse standing modes, which manifested themselves as oscillations in Doppler velocity in an emission line sensitive to hot plasma of $6 \mathrm{MK}$. Although the evidence presented suggests that the coronal oscillations observed in hot flare lines are slow magneto-acoustic modes, instruments with high spatial resolution e.g. the Atmospheric Imaging Assembly (AIA) on the Solar Dynamics Observatory (SDO) (Lemen et al. 2011) that are able to make observations in hot coronal lines, may be able to confirm this or to provide a new interpretation for such hot oscillations.

The AIA has seven extreme ultraviolet (EUV) bandpasses (Fe XVIII (94 ̊), Fe VIII/XXI (131 ̊), Fe IX (171 ̊), Fe XII/XXIV (193 ̊̊), Fe XIV (211 A), He II (304 ̊), and 
Fe XVI (335 ̊)) spanning a temperature range between approximately $1 \mathrm{MK}$ and $11 \mathrm{MK}$ allowing a wide range of observations to be made. However, to date, TLOs have only been observed in the cooler coronal lines corresponding to coronal temperatures of approximately 1-2 MK. Transverse loop oscillations (e.g. Nakariakov et al. 1999; Aschwanden et al. 1999, 2002; Aschwanden \& Schrijver 2011; Wang \& Solanki 2004; Hori et al. 2005, 2007; Verwichte et al. 2009, 2010; McIntosh et al. 2011; Mrozek 2011; White \& Verwichte 2012; Wang et al. 2012) can be either vertically or horizontally polarised and are interpreted as the magneto-hydrodynamic kink mode (Edwin \& Roberts 1983; Nakariakov et al. 1999; Goossens et al. 2009) observed in the corona as the perturbation of the axis of density enhanced loops.

To date, there have been few observations of TLOs displaying higher harmonics. The first observation of higher harmonics in a coronal loop arcade was made by Verwichte et al. (2004). Here both the fundamental mode and the second harmonic were observed in a wavelet spectrum and the amplitude signatures were found to be consistent with two different periodicities. This first observation was followed by analytic and numerical studies of the density stratification in coronal loops (Andries et al. 2005a,b; McEwan et al. 2006; Dymova \& Ruderman 2006). In particular, the density stratification inside coronal loops is related to the ratio of the fundamental period $\left(P_{1}\right)$ to the period of the second harmonic $\left(P_{2}\right)$. Another observation of higher order harmonics in TLOs was made by De Moortel \& Brady (2007) for which a repeat analysis was conducted by Van Doorsselaere et al. (2009). This spatially resolved TRACE observation allowed three periodicities to be detected (which were identified as the fundamental mode and both the first and second harmonics). A third example of a higher harmonic TLO (Van Doorsselaere et al. 2007) identified both the fundamental mode and the first harmonic, which were detected in the signal residues.

Questions arising from the study of higher order harmonics include (1) why are the majority of observed TLOs excited as the fundamental mode and (2) what is the excitation method responsible for higher order harmonics? AIA/SDO provides new insights into coronal phenomena by increasing both the time and spatial resolutions. As coronal features such as TLOs are studied in ever greater detail (White \& Verwichte 2012), signatures of higher harmonics may become easier to observe. The SDO may also provide further opportunities to study the exact nature of oscillations in hot coronal loops.

In this paper, we present the first analysis of a higherharmonic transverse oscillation in a hot loop observed with AIA/SDO. This observation is particularly interesting as the loop oscillates with a dominant higher-harmonic vertical mode and shows little evidence of a fundamental mode. Furthermore, it is only observed in the $131 \AA(\sim 10 \mathrm{MK})$ and $94 \AA(\sim 6.3 \mathrm{MK})$ bandpasses. This is an important observation as there are few spatially observed TLOs and none that have been observed in hot bandpasses.

The paper is organised as follows: Sect. 2 provides an overview of the event, Sect. 3 describes our time series analysis of the loop oscillations, Sect. 4 discusses the intensity variations of the loop, Sect. 5 presents the results of the loop-geometry and loop-mode modelling, our discussion is presented in Sect. 6 and the paper conclusions are presented in Sect. 7.

\section{Event overview}

The event occurred between 12:10:09 and 13:09:57 UT on the 3 November 2010 and has previously been studied in the context
Table 1. Sequence of events in active region NOAA 11121 on November 3, 2010.

\begin{tabular}{lcc}
\hline \hline Event type & Time $(\mathrm{UT})$ & Instrument \\
\hline CME & $12: 13: 21$ & AIA \\
1 MK loop oscillations & $12: 13: 36$ & AIA 171 $\AA$ \\
Flare peak & $12: 14$ & RHESSI 25-50 keV \\
Hot loop oscillation & $12: 16: 33$ & AIA 131 \\
Flare peak & $12: 17: 30$ & RHESSI 3-6 keV \\
Flare peak & $12: 21$ & GOES-14 1-8 \\
\hline
\end{tabular}

of the coronal mass ejection (CME) (Reeves \& Golub 2011; Cheng et al. 2011; Bain et al. 2012), Kelvin-Helmholtz instability on the flank of the CME (Foullon et al. 2011), and transverse coronal loop oscillations (White \& Verwichte 2012). During this event, a CME associated with a C4.9 class flare observed by the Geostationary Operational Environmental Satellite (GOES), peaking at 12:21 UT, was observed. A large loop oscillating exclusively in the $131 \AA$ (Fe VIII/XXI) and $94 \AA$ (Fe XVIII) bandpasses, appears near the south-east solar limb south of the CME ejecta after the peak of the hard X-ray flare. The left panel of Fig. 1 shows an image of the event in the $131 \AA$ bandpass where a loop structure can be seen off the limb below the flare/CME site. The $131 \AA$ emission corresponds to plasma temperatures around $11 \mathrm{MK}$ and just under $1 \mathrm{MK}$. The $94 \AA$ bandpass corresponds to temperatures around $9 \mathrm{MK}$ and $1 \mathrm{MK}$. As this loop is not observed in any other of the AIA bandpasses e.g. $171 \AA$ ( $\sim 1 \mathrm{MK})$, we expect this loop to be filled with hot plasma.

The right panel of Fig. 1 shows a difference image in the $131 \AA$ bandpass, which highlights the oscillating loop. During the observation, the loop appears to oscillate in a harmonic overtone, i.e. there is a node along the loop around which the legs of the loop oscillate in anti-phase to one another. The difference image suggests that there is a node at a position of approximately $\left(955^{\prime \prime} \mathrm{E}, 450^{\prime \prime} \mathrm{S}\right)$ relative to the Sun centre about which the loop oscillates in anti-phase. We introduce a projected length coordinate $s$ that runs along the loop in the image from the northern to the southern footpoints (as seen in left panel of Fig. 1). Figure 2 shows time-distance images from cuts made along the loop (see Fig. 1 left for the cut locations) and a clear phase change is observed with increasing $s$. Figure 2 suggests that the node must lie between approximately $s=103 \mathrm{Mm}$ and $s=114 \mathrm{Mm}$. The node is situated on the southern leg close to the loop top as observed in the images. This further suggests that, if this is a second harmonic, the southern loop footpoint is situated behind the solar limb since a node would be expected at the loop top. Because the loop is likely to be partly occulted by the solar disk, using the height above the limb to estimate the radius and hence length of a circular loop will lead to a gross underestimation of the real loop length.

Figure 3 shows when the loop oscillation occurred with respect to the flare observed in both hard and soft X-rays (by the Reuven Ramaty High Energy Solar Spectroscopic Imager (RHESSI) and the GOES satellites), in addition to the CME. Table 1 lists the events and the time at which they were observed. The peak of the RHESSI hard X-ray flare $(25-50 \mathrm{keV})$ occurs at 12:14 UT, which is just after the start of the oscillations in the $171 \AA$ bandpass. The soft X-ray flare peaks at 12:17:30 (RHESSI 3-6 keV) and 12:21 UT (GOES 1-8 ), where both times are after the start of the $131 \AA$ loop oscillation. The loop appears in the EUV images around 12:16:09 UT and is no longer visible by 12:50:09 UT, although the loop fades after 

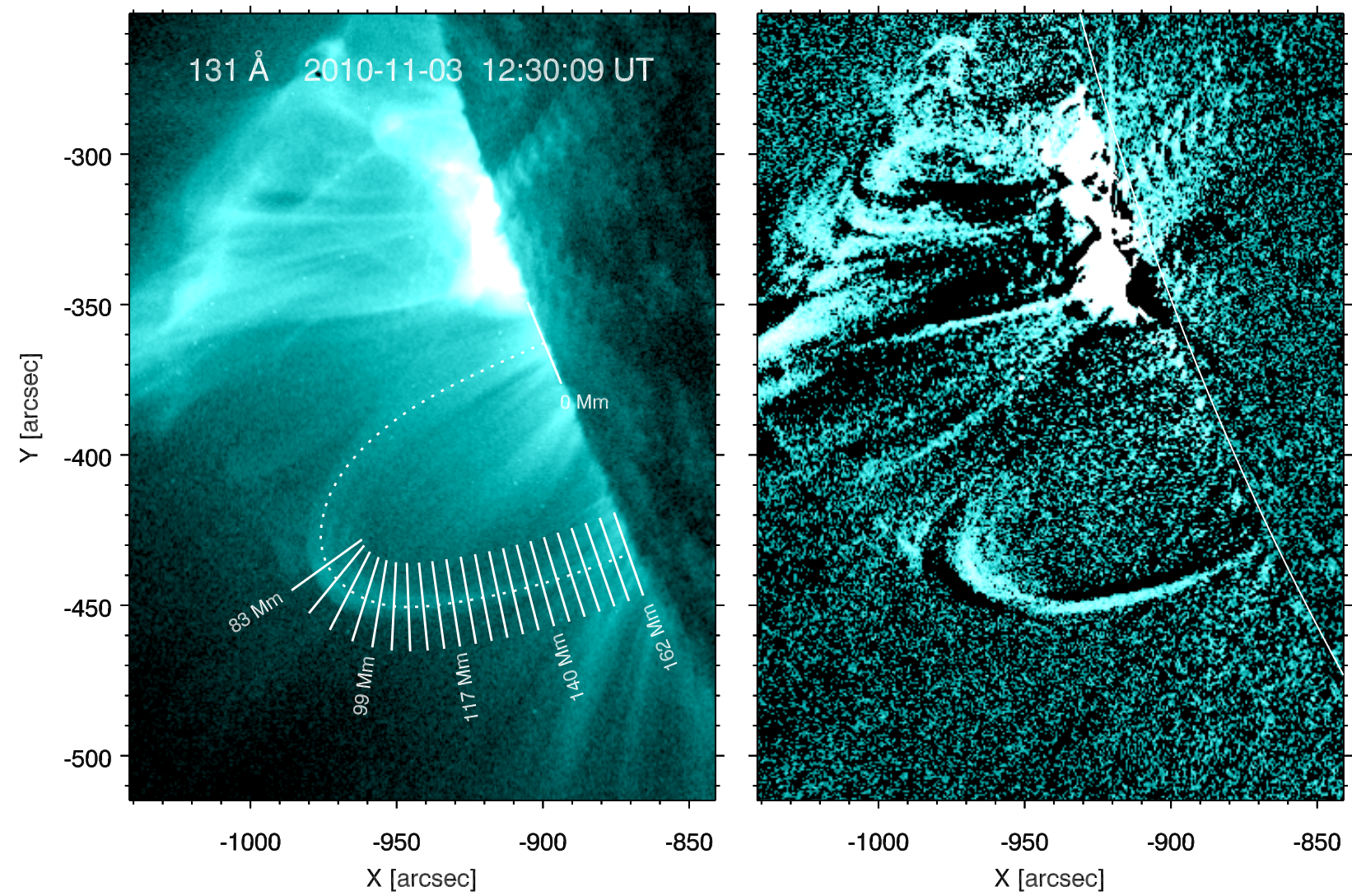

Fig. 1. AIA/SDO $131 \AA$ A image of the November 3 event. Left: image of the event outlining the position of the hot loop below the CME ejecta. Cuts taken to analyse the oscillation are displayed and are separated by $\sim 4 \mathrm{Mm}$. The distance co-ordinate $s$ begins at the top leg and ends at the bottom leg. Right: a difference image highlighting the loop oscillation, which was produced by subtracting the image taken at 12:20:57 from the one at 12:22:57 UT. The solar limb is indicated by the solid white line.

approximately 12:40 UT. We do not see any evidence of hot downflows. In addition, we do not observe the loop appearing in colder coronal bandpasses, though there is much line-of-sight confusion that prevents us from being absolutely certain. There is evidence of cool downflows, visible in $304 \AA$, that may be located in part of the northern leg of the loop which is not visible in the hot coronal bandpasses.

\section{Time series analysis}

Our time series analysis of the loop is performed using the same method as employed in White \& Verwichte (2012). Here cuts are made along the loop, in the direction perpendicular to the loop axis, to extract time-distance images. Any transverse oscillations present will show up in these images, with the clearest results being attainable from cuts that are parallel to the projected oscillation displacement. Time series analysis is performed for the cuts displayed in Fig. 1 (left). Results for the northern loop leg as observed in the images are not obtained because of the low loop contrast with the background plasma. This means that oscillations are not observed in the time-distance images for this loop leg. The angle of the cuts is varied to check that these cuts are the best, i.e. that they are parallel to the projected displacement direction so that they give the clearest images with the largest oscillation amplitude. A two-dimensional (2D) wavelet transform using the Mexican hat wavelet (Witkin 1983) is used to improve the contrast of the images and distinguish multiple loop strands from one another. A Levenberg-Marquardt leastsquares fitting method (Markwardt 2009) is used to fit a damped cosine function to the background-subtracted time series, which in all cases is estimated by eye. The reliability of the time series curves is checked by plotting a marker on the AIA images corresponding to the loop location and playing the images as a movie. The marker can then be observed to follow the loop as it oscillates. A time series analysis is only performed for cuts taken from the $131 \AA$ bandpass owing to the low contrast of the loop in the $94 \AA$ bandpass, which makes the extraction of oscillatory information unreliable. Figure 4 shows an example of the time series analysis method for a cut taken at $s=144 \mathrm{Mm}$. The upper panel of Fig. 4 shows a time-distance image displaying a damped oscillation, which is highlighted further in the middle panel after the application of the wavelet transform. A displacement time-series is then estimated by eye as shown by the dashed line. This analysis is performed for all cuts shown in Fig. 1, except for six cuts between $96 \mathrm{Mm}$ and $114 \mathrm{Mm}$ where a node is expected and the oscillation in the cuts is not easily observable. In six of the time-distance images, two loop strands are observed. In these cases, oscillatory information for both strands is extracted. The lower panel shows an example of the backgroundsubtracted time series that is fitted with a damped cosine curve to determine the oscillation parameters of the period $(P)$, damping time $(\tau)$, amplitude $(\xi)$, and phase $(\phi)$. Figure 5 shows the resulting loop parameters $P, \tau, \phi$, and $\xi_{0}$ with respect to $s$ along the loop. The triangles show the results for the second loop strand (observed in six of the time-distance images). The error bars display the errors returned by the fitting routine. Displayed in the figures for $\tau$ and $P$ are the mean and standard deviations for the first strand. The time series analysis for the first (second) strand 


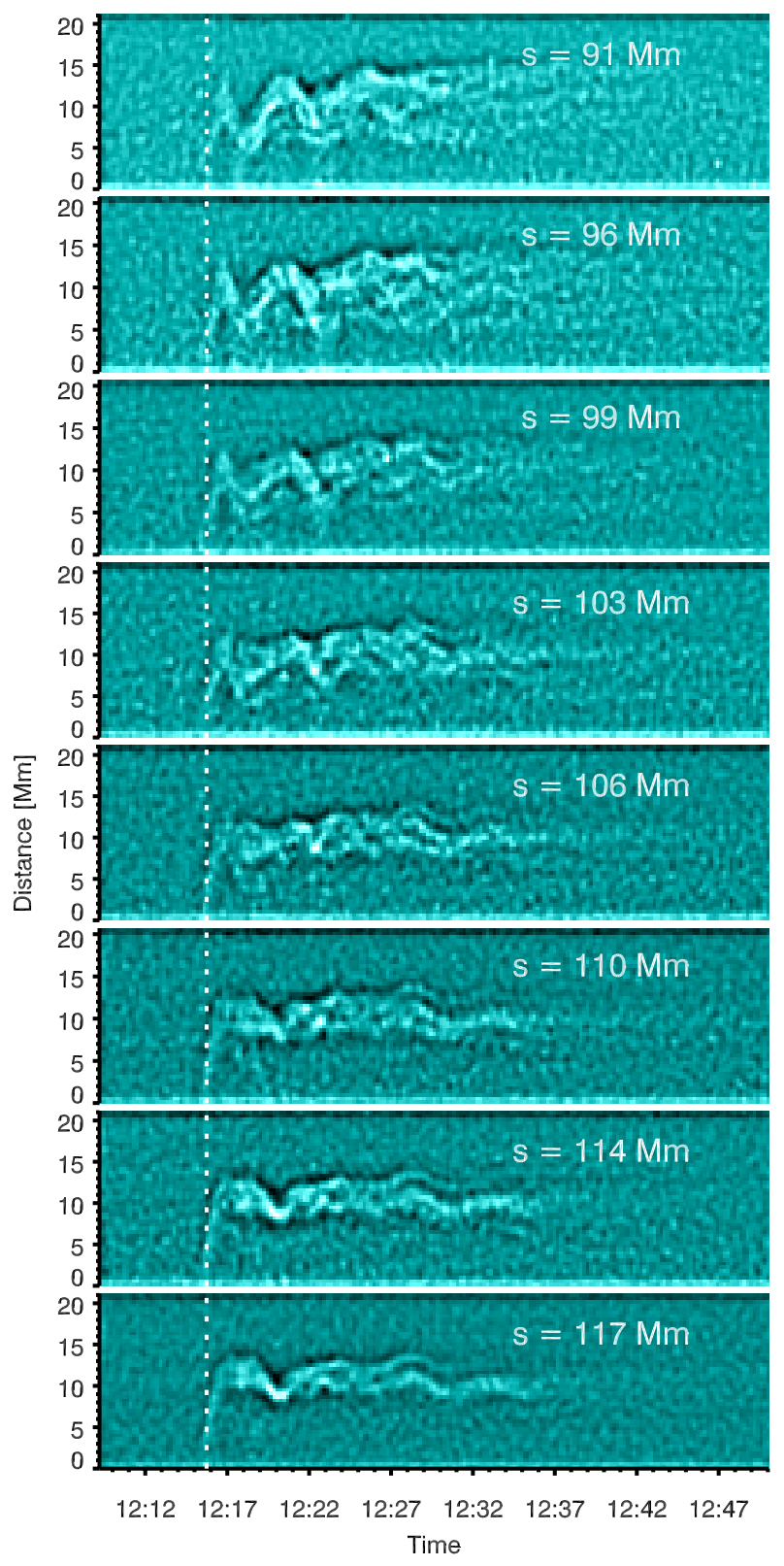

Fig. 2. Time-distance images extracted from cuts taken along the loop to analyse the oscillation. The cuts span the region where it is expected a node lies. The loop oscillations are observed to undergo a $180^{\circ}$ shift in phase in the region between $s=103$ and $s=114 \mathrm{Mm}$. The vertical dotted line indicates the reference time of 12:16:33 UT.

gives a period of $P=302 \pm 14 \mathrm{~s}(291 \pm 9 \mathrm{~s})$ and a damping time of $\tau=306 \pm 43 \mathrm{~s}(487 \pm 125 \mathrm{~s})$. The errors were determined from the standard deviation in the results.

The upper panel of Fig. 5 shows that $\phi$ undergoes a $180^{\circ}$ phase shift along the loop, which is a clear signature that what we observe is a harmonic overtone. We would expect the amplitude of the oscillation to decrease as we go towards the node position, although the oscillations in the cuts close to the node appear messy in the time-distance images with overlapping loop strands. Therefore, reliable time-series information around the node position is not obtained. Figure 2 shows time-distance slices close to the node point (between approximately $103 \mathrm{Mm}$ and $114 \mathrm{Mm}$ ). This figure shows that the oscillations do not completely die away but that the amplitude does decrease and oscillations are harder to extract from the images. It may be that there are still some amplitude signatures from the fundamental mode
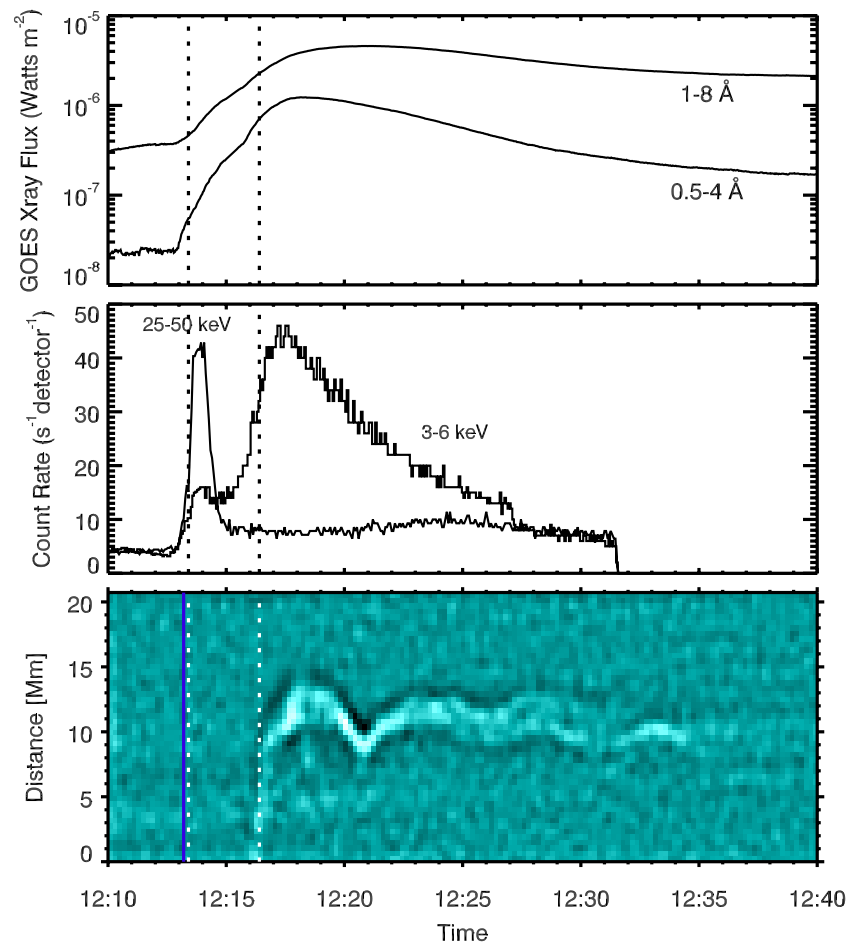

Fig. 3. Upper: GOES (1-8 and $0.5-4 \AA)$ soft X-ray flux. Middle: RHESSI (3-6 and 25-50 keV) X-ray flux. The 25-50 keV curve has been scaled by 0.25 . Lower: an example time-distance cut of the $131 \AA$ loop oscillation. Times of different events are indicated. Blue solid line: approximate start of CME eruption at 12:13:21 UT. Dotted lines indicate the reference times of the oscillations, 12:13:36 UT for the $171 \AA$ loops from White \& Verwichte (2012) and 12:16:33 UT for the $131 \AA$ loop.
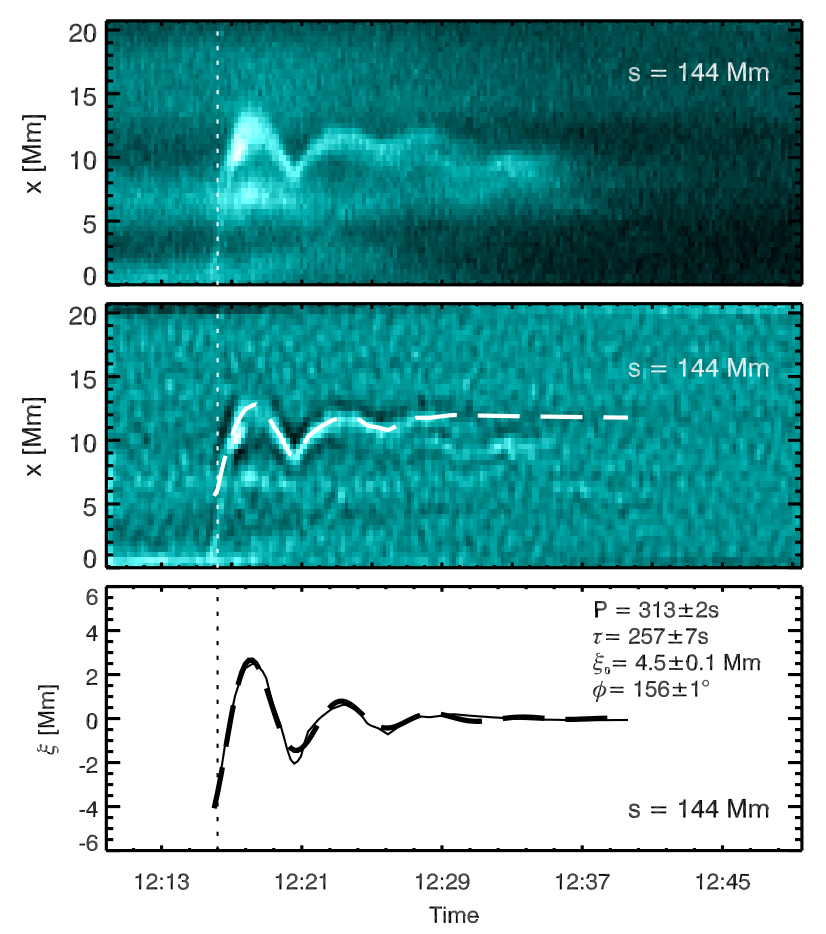

Fig. 4. Upper: time-distance image extracted from a cut at $s=144 \mathrm{Mm}$. Middle: a 2D Mexican hat wavelet transform has been applied to the extracted image to highlight the oscillations present. Lower: the time series was de-trended and fitted with a damped cosine function to give information on the amplitude, period, damping time, and phase. The vertical dotted line indicates the reference time of 12:16:33 UT. 
R. S. White et al.: Transverse vertical oscillation during the formation of a hot post-flare loop
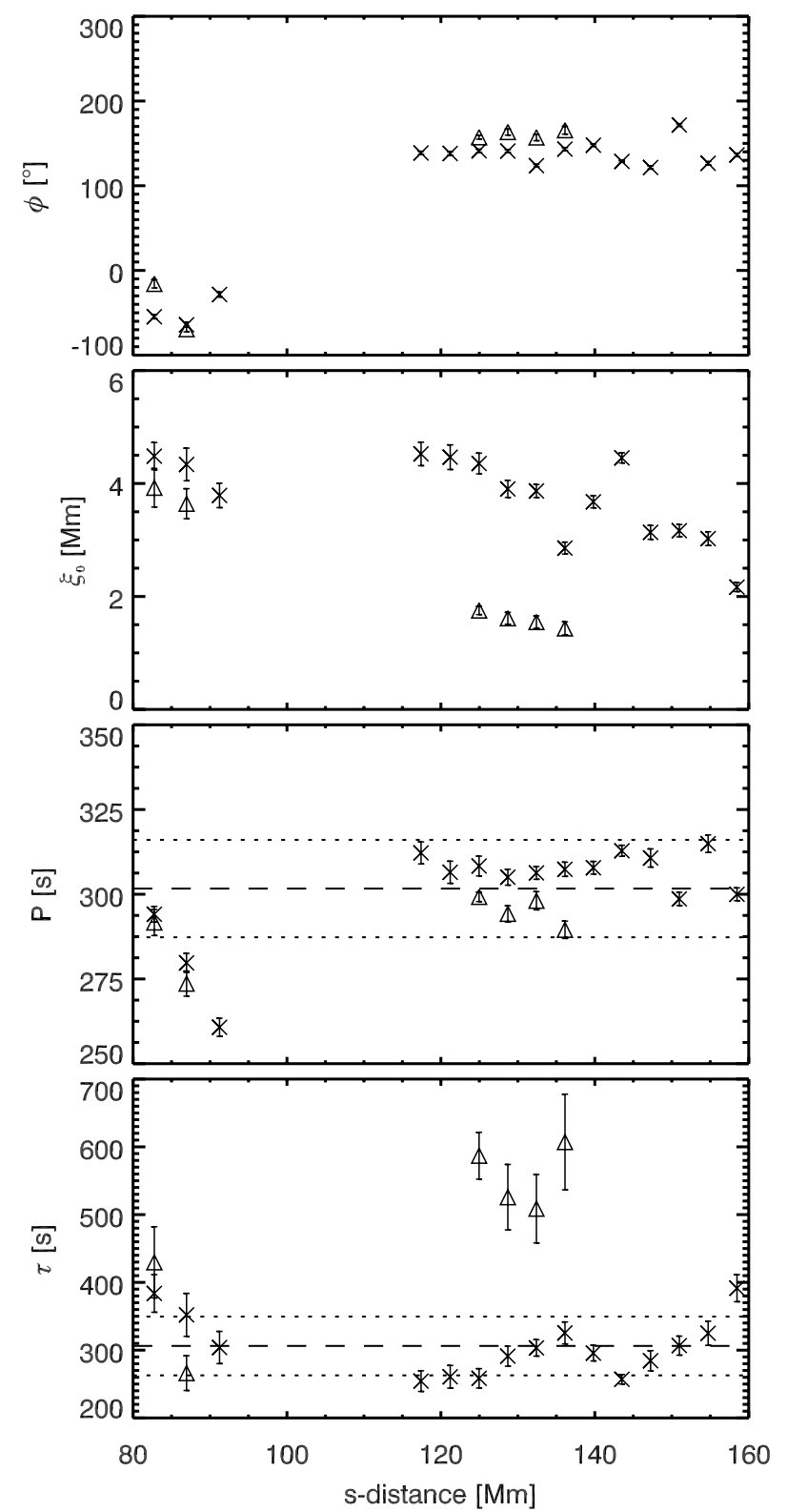

Fig. 5. Oscillation parameters, phase $(\phi)$, projected displacement amplitude $(\xi)$, period $(P)$, and damping time $(\tau)$ plotted as a function of $s$ along the loop. The triangles show the results obtained for the second loop strand observed in six of the time-distance images. The error bars show the errors given by the fitting technique. The horizontal dashed line in the bottom two plots indicate the mean value of $P$ and $\tau$ for the first loop strand and the horizontal dotted lines indicate the standard deviation. The reference time is 12:16:33 UT.

(which would be a maximum at the loop top, i.e. a maximum at the second harmonic node) or other higher harmonics that do not allow the amplitude to go to zero at this point. The observations indicate that the southern footpoint is situated behind the solar limb. The amplitude does decrease as would be expected towards the southern footpoint. The period and phase of the second strand (indicated by the triangles) are consistent with those of the first strand. The periods and damping times are mostly within one standard deviation of the mean.

\section{Loop intensity}

To investigate any variations in the intensity of the loop oscillation, intensity is extracted at each point along the displacement

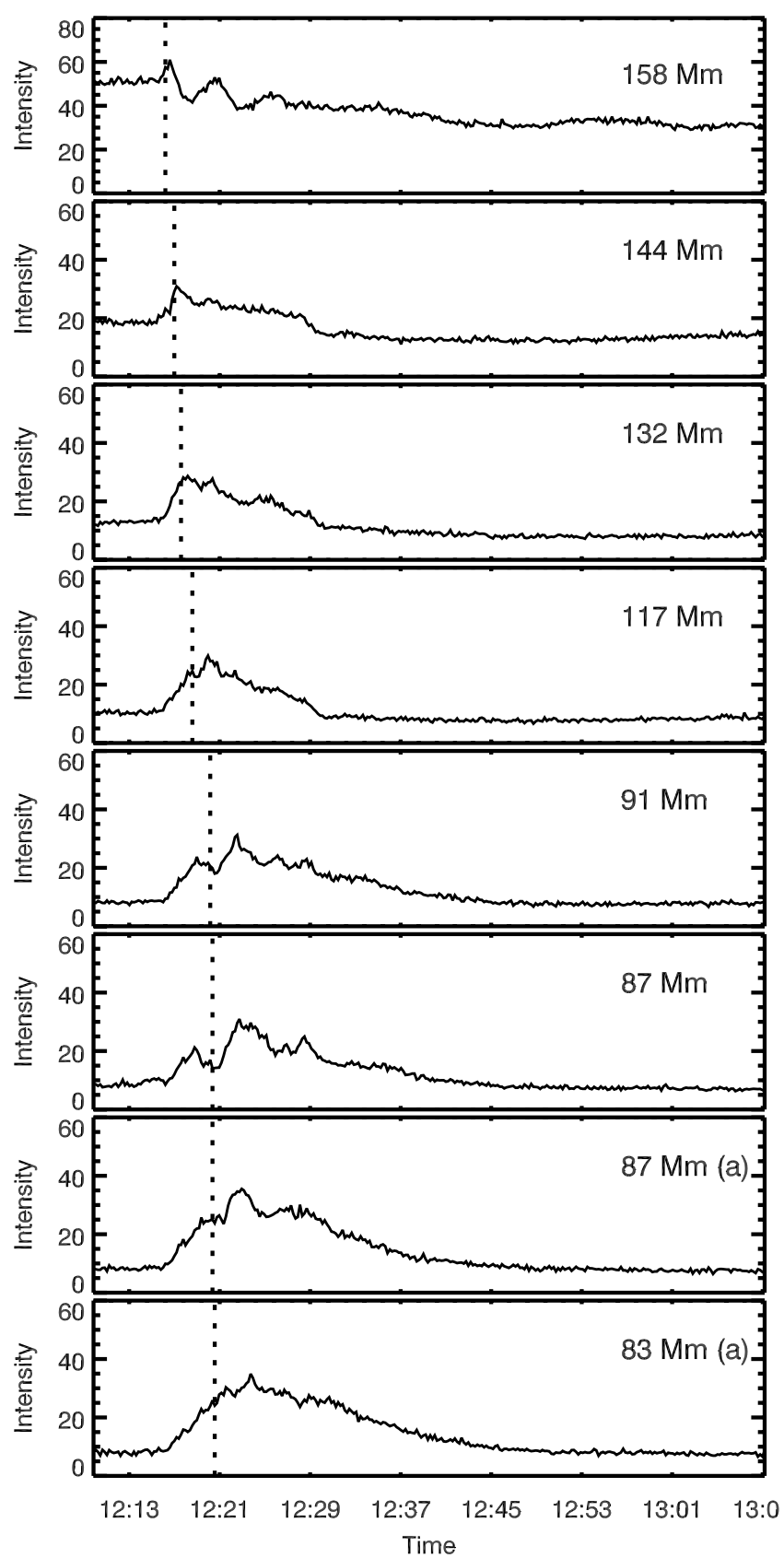

Fig. 6. Intensity along the loop oscillation path extracted from timedistance images by taking the intensity at each point along the displacement time curves for eight values of $s$ along the loop. (a) Indicates that the intensity is extracted from the second strand which was observed in six of the time-distance images, the results of which are represented as triangles in Fig. 5. The vertical dotted line represents the position in time of the linear fit to the intensity shown in Fig. 8.

time-series for each of the cuts. Figure 6 shows examples of the extracted intensity as a function of time for different values of $s$, and at a first glance resembles the signatures of flare light-curves. The intensity rises sharply and then diminishes more slowly. It also seems to diminish more slowly towards the loop top compared to lower down the southern loop leg towards the footpoint. Oscillatory signatures can also be seen in the extracted intensity, particularly at $s=158 \mathrm{Mm}$.

The oscillations present in the intensity are investigated further by de-trending the intensity and fitting a damped cosine curve using the same method as for the time series analysis. 

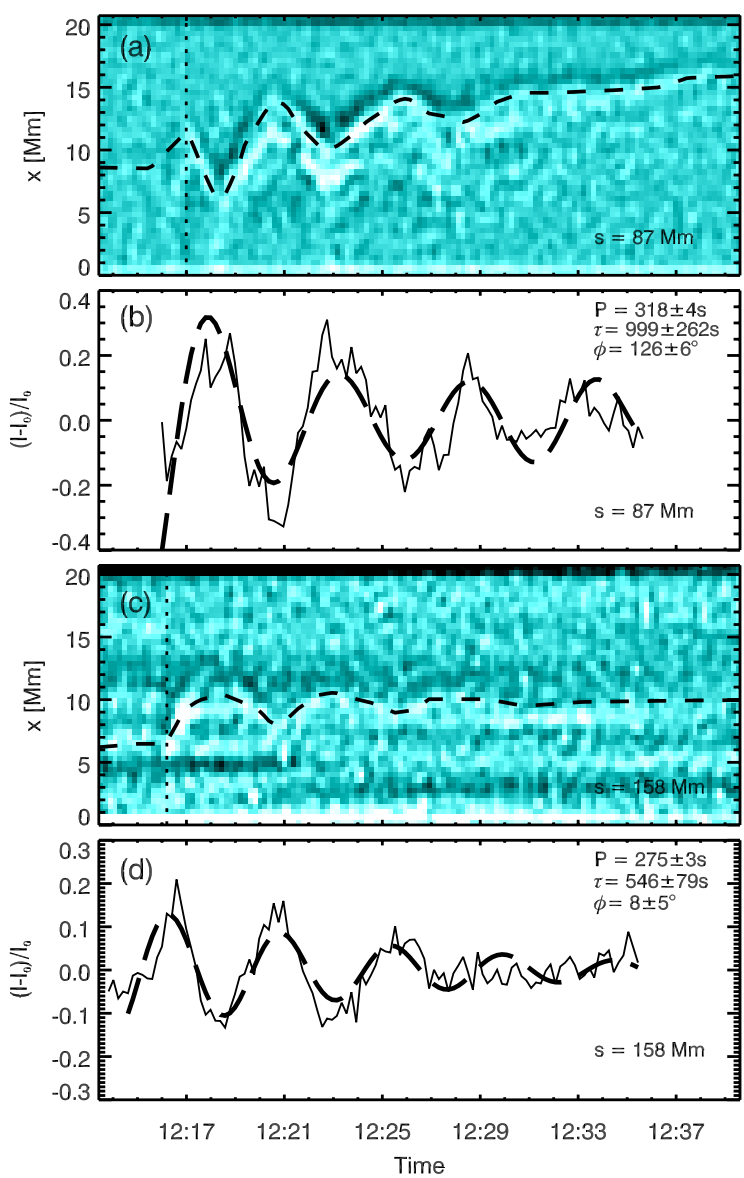

Fig. 7. Analysis of the intensity time-series for cuts at $s=87 \mathrm{Mm}(\mathbf{a}, \mathbf{b})$ and $s=158 \mathrm{Mm}(\mathbf{c}, \mathbf{d})$; a) and c) show the time-distance images with the oscillation time-series overplotted. The vertical dashed lines indicate the start of the oscillation; b) and d) show the intensity time-series extracted from the time-distance images. The thick dashed line is the damped cosine fit to the intensity.

Figure 7 shows this analysis performed for cuts located at $s=$ $87 \mathrm{Mm}$ and $s=158 \mathrm{Mm}$ (a damped cosine could not be fitted to the de-trended intensity from cuts at any other values of $s$ ). Here the location of the time series has been overplotted on the time-distance images (panels a, c) with the extracted intensity time-series displayed below (panels b, d). The intensity time-series is background-subtracted and then normalised by the background. Panels $b$ and $d$ show significant intensity variations with respect to the background intensity, between $10 \%$ and $30 \%$ for the peaks of the oscillations. The periods of both these intensity oscillations, $318 \pm 4 \mathrm{~s}$ and $275 \pm 3 \mathrm{~s}$, lie just outside the displacement time-series period of $302 \pm 14 \mathrm{~s}$ and hence are broadly consistent. The intensity oscillations are approximately in anti-phase with the displacement time-series analysis for their respective cuts. This means that as the loop displacement is upwards (downwards), the intensity decreases (increases).

We also investigate the intensity along the loop axis as a function of time as shown in Fig. 8. The cut has a width of 81 pixels (48.6") which ensures that the whole of the loop oscillation is captured at each time. The time-distance cut is averaged over this width to increase the signal to noise. The raw time-distance image is averaged in time and then this average intensity is subtracted from the raw time-distance image. This helps to highlight any features that may be present. Figure 8 shows that intensity first appears near the southern footpoint and
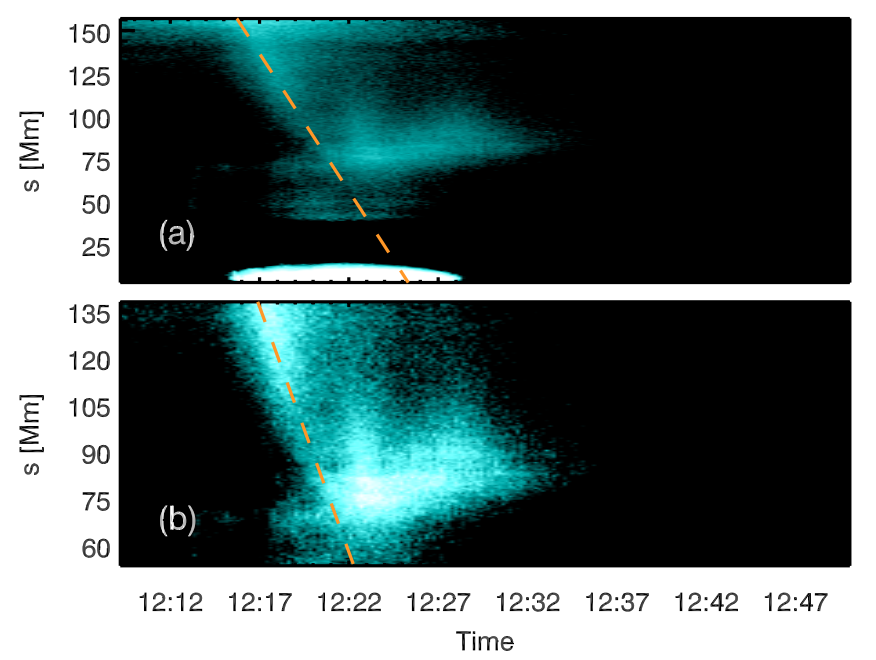

Fig. 8. Intensity along the loop plotted as a function of time. The intensity averaged in time has been subtracted from each time slice. a) The linear trend (orange dashed line) gives a projected speed of $212 \mathrm{~km} \mathrm{~s}^{-1}$. b) Zoomed-in version of the top figure giving a projected speed of $206 \mathrm{~km} \mathrm{~s}^{-1}$. This speed can be approximately corrected for the projection using the $3 \mathrm{D}$ estimate of the loop from the STEREO-B/SDO comparison to give a speed of approximately $470 \mathrm{~km} \mathrm{~s}^{-1}$, close to the sound speed of $500 \mathrm{~km} \mathrm{~s}^{-1}$ at $11 \mathrm{MK}$.

then "flows" along the loop towards the top. The loop has then almost disappeared by 12:40 UT. A linear trend is fitted to the intensity brightening (dashed orange line in Fig. 8) with the gradient of the intensity front giving projected speeds of $212 \mathrm{~km} \mathrm{~s}^{-1}$ (panel a) and $206 \mathrm{~km} \mathrm{~s}^{-1}$ for a shorter path (panel b).

\section{Loop geometry and mode modelling}

Comparing the loop observed from the viewpoint of SDO with that from another viewpoint, such as the Solar Terrestrial Relations Observatory (STEREO), can allow the 3D loop geometry to be estimated. Loop points are chosen in the STEREO-B viewpoint with the Extreme Ultraviolet Imager (EUVI) (Howard et al. 2008), which are then projected onto the other SDO/AIA viewpoint assuming a planar loop model. By comparing the projected loop points for the two viewpoints with an imposed (userdefined) inclination angle $(\theta)$ with respect to the photospheric normal, $\theta$ can be constrained. In this observation, the loop is only seen in the $131 \AA$ and $94 \AA$ AIA bandpasses and not observed in the EUVI channels, although possible footpoint locations are identified in the EUVI images. By comparing the geometry of the CME (observed in both the cooler and hotter bandpasses) in both AIA and EUVI and knowing the position of the loop relative to the CME in the AIA images, an estimate of the geometry of the hot loop is obtained. Figure 9 shows the estimated loop geometry from both the STEREO and SDO viewpoints. Since the loop is not observed in the EUVI images and its geometry is inferred from that of the CME, this geometry is the most likely one and not an absolute model of the loop. Using this technique, an optimal inclination angle of $-6^{\circ}$ and a loop length of $466 \mathrm{Mm}$ is found. Figure 9 shows that the 3D geometry estimate does not precisely match the loop observed in the AIA images. This is to be expected since, although reliable estimates for the footpoint locations are found, it is difficult to follow a loop path in the STEREO images. In addition a planar loop model is assumed, which may not be an accurate assumption in this case. 

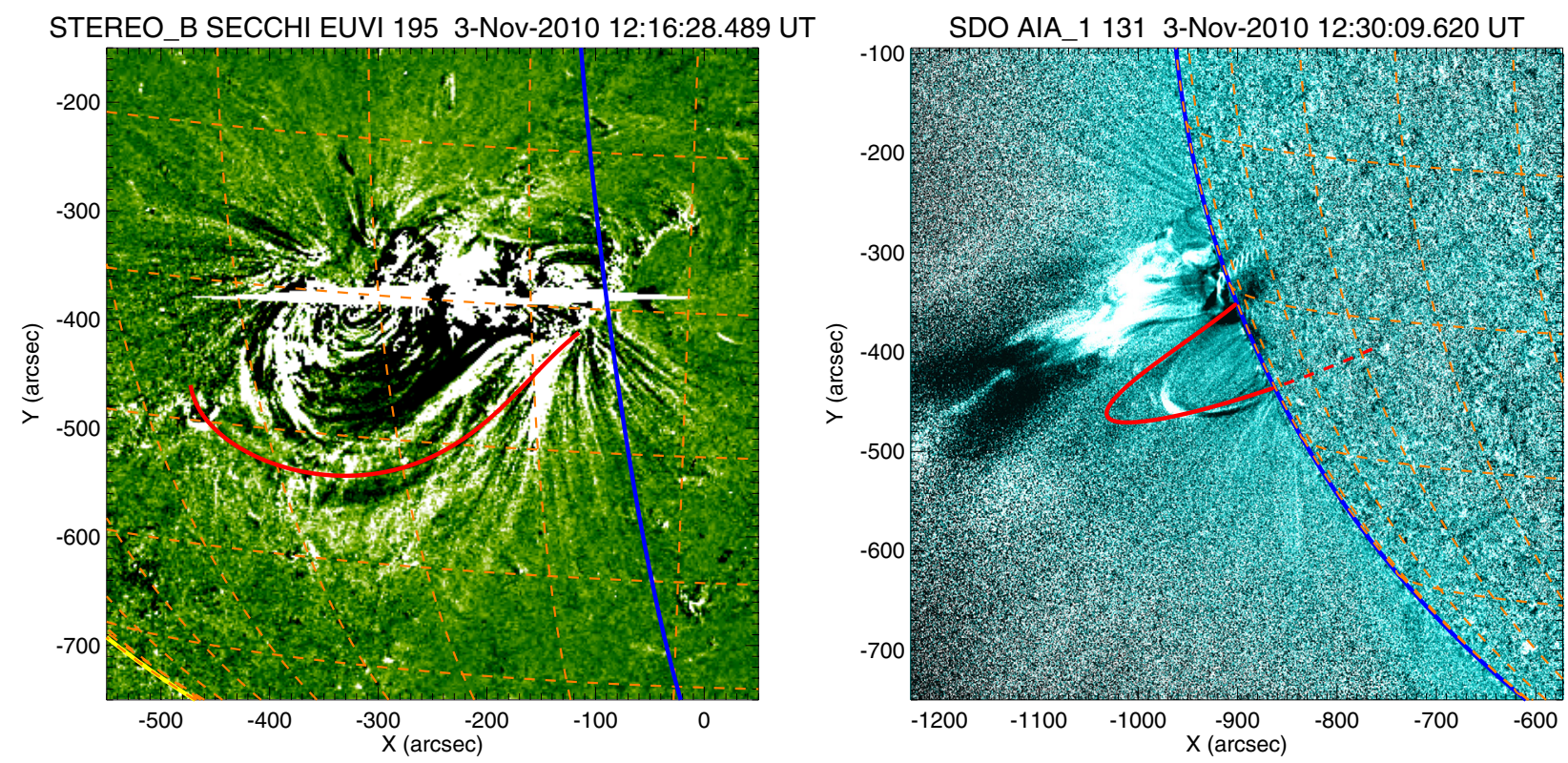

Fig. 9. Left: STEREO-B EUVI $195 \AA$ difference image (at the time indicated with respect to 12:11:28.475 UT). Right: SDO AIA $131 \AA$ image ratio (at the time indicated with respect to a previous image at 12:18:57.620 UT). Overlaid are the loop trace (red thick line with dashed lines for portion behind the limb in right panel), the SDO limb (blue) and heliospheric grid lines spaced by 10 degrees (orange dashed lines).

With an estimate of the 3D geometry, different modes of transverse oscillations are modelled to see whether any of them match the observed loop motion. The loop is modelled for the AIA viewpoint and is uniformly filled to a radius of $3 \mathrm{Mm}$ and a contrast of 10 with the background. We refer to White $\&$ Verwichte (2012), Sect. 5.1, for details of the modelling procedure. The first three harmonics of both the horizontally and vertically polarised kink modes are modelled with an amplitude of $4.7 \mathrm{Mm}$. Figure 10 shows the difference images for each of the modelled modes. In the AIA difference image (right panel of Fig. 1), one node is clearly observed on the southern leg of the loop, although the northern leg is not observed because of the poor contrast. The modelled modes that most closely match the observations are the $n=2$ and $n=3$ vertically polarised modes (Fig. 10b, c). The observation along with the forward modelling suggests that this loop is oscillating as a vertically polarised second or third harmonic. If it is a third order harmonic, there should be a node situated on the northern leg, which is not visible as the contrast between the loop and the background plasma diminishes.

\section{Discussion}

Vertically polarised TLOs were previously reported by Wang \& Solanki (2004), Mrozek (2011), and Aschwanden \& Schrijver (2011). Key questions from this observation are: (1) what are the conditions and/or mechanisms behind the excitation of this oscillation?; (2) why is a vertical harmonic overtone selected as the mode of oscillation?; and (3) why is the loop only observed in the hot bandpasses?

\subsection{Loop generation and excitation}

Both the CME ejecta and the loop are observed in the $94 \AA$ and $131 \AA$ bandpasses suggesting that they are directly linked to one another and that the loop is generated by the CME. Figure 11 shows a strand originating from the direction of the
CME towards the location of the loop just before the loop appears at about 12:16 UT. The position of the loop inferred from Fig. 1 is indicated by the dotted line. The strand appears to link the location of the top of the loop, as observed with AIA, to the CME. This may indicate that the loop is directly formed by the CME as a result of reconnection processes forming and releasing field lines that are then filled with hot plasma at the footpoints.

Figure 8 shows that hot plasma is injected into the loop from the southern footpoint, allowing it to become visible in the hot AIA channels. This asymmetric plasma heating of the loop has a projected speed of about $210 \mathrm{~km} \mathrm{~s}^{-1}$. Using the 3D loop geometry found in Sect. 5, this speed can be approximately corrected for the projection since in the AIA image the distance to the loop top is $80 \mathrm{Mm}$, whereas in the modelled loop it is about $180 \mathrm{Mm}$. This gives a corrected speed of approximately $470 \mathrm{~km} \mathrm{~s}^{-1}$, which is close to the sound speed of $500 \mathrm{~km} \mathrm{~s}^{-1}$ for an $11 \mathrm{MK}$ plasma. We might then expect the hot plasma to cool in time and become visible in the cooler AIA bandpasses, but this is not observed. It may be that the plasma cools on a timescale that is much shorter than can be observed owing to a thermal instability and becomes directly visible in cooler bandpasses such as $304 \AA$. We conclude that the upflow is consistent with explosive chromospheric evaporation and there is some evidence at $304 \AA$ of gentle, cool condensating downflows (e.g. Fisher et al. 1985; Milligan et al. 2006; Milligan \& Dennis 2009).

The observed hot plasma injected can be expected to excite a fundamental slow magneto-acoustic oscillation in the loop (Wang et al. 2003b; Nakariakov et al. 2004; Tsiklauri et al. 2004). For a loop length of $L=466 \mathrm{Mm}$ and sound speed of $C_{\mathrm{s}}=500 \mathrm{~km} \mathrm{~s}^{-1}$, we found a period of approximately $30 \mathrm{~min}$, which is longer than the duration of the observation when the loop is effectively visible. Therefore, we found no evidence of a slow wave in this loop.

The excitation time of the transverse loop oscillation corresponds to the gradual phase of the flare rather than the earlier impulsive phase of the flare (see Fig. 3). A possible explanation for the excitation of this TLO may therefore be as follows. 

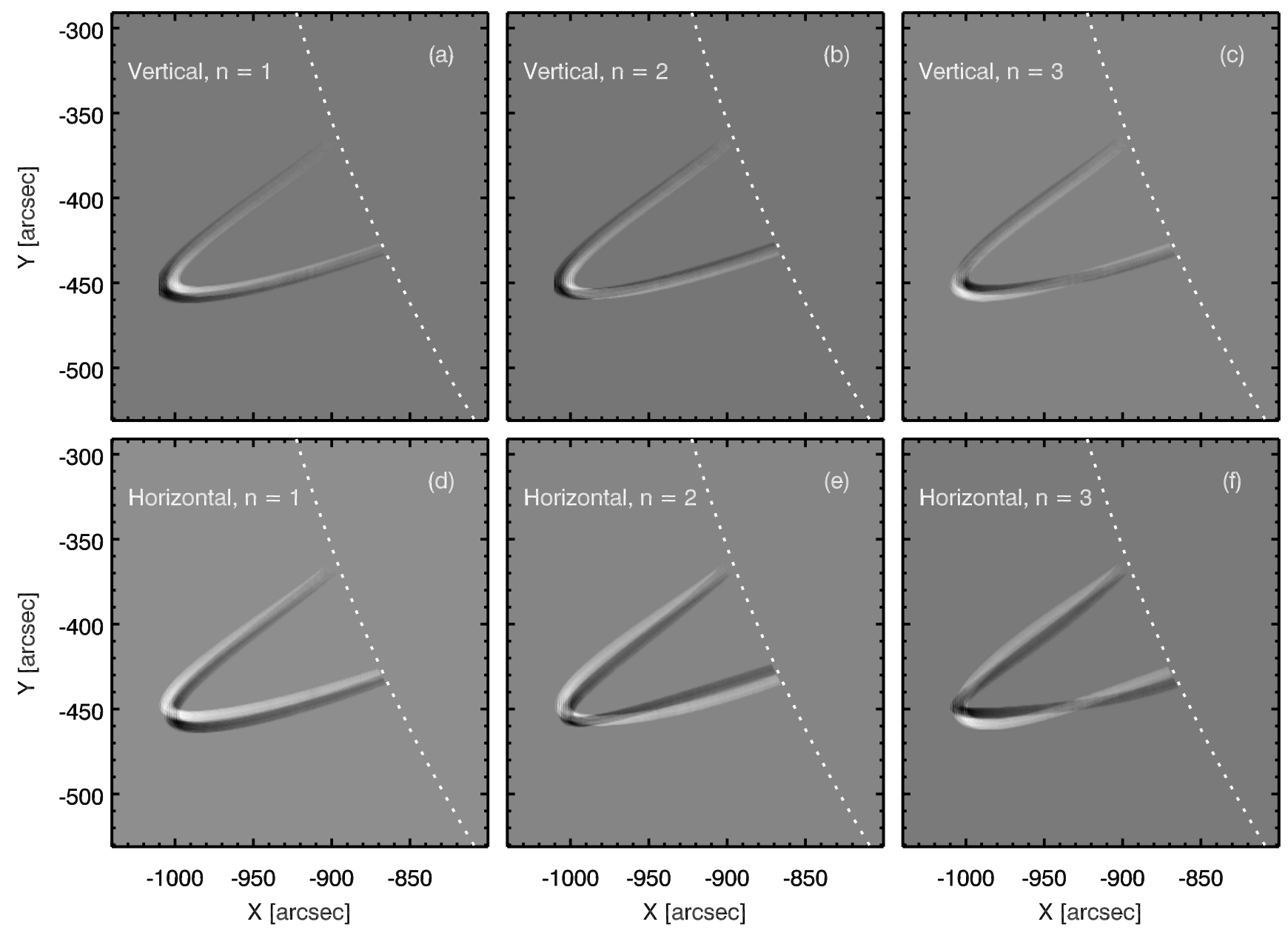

Fig. 10. Difference images of the modelled horizontally and vertically polarised transverse modes in the AIA viewpoint. Top: vertically polarised; a) first harmonic; b) second harmonic, c) third harmonic. Bottom: horizontally polarised; d) first harmonic, e) second harmonic, f) third harmonic. The dotted line indicates the solar limb.

Field lines form from a reconnection process creating a loop structure that is held at the loop top. The top is then released (or even pushed down) causing the loop to oscillate as a vertical TLO. This is a more likely scenario than the loop being hit by the blast wave (as for the TLOs observed in $171 \AA$ ) as the loop appears to be released from the top rather than be hit from the side. The 3D forward-mode modelling (Fig. 10) also supports this interpretation, suggesting that the loop is a vertical mode oscillating as a second or third harmonic. The numerical simulations of a CME eruption from an anemone active region by Lugaz et al. (2011) shows a two-stage reconnection process that includes the formation of post flare loops (see their Fig. 5). Although there are differences between the numerical simulation and this observation, a comparison shows that reconnection could play an important role in the formation and wave excitation of the hot loop.

Numerical simulations of vertically polarised modes have been conducted where a pulse is generated below the loop, often modelled in slab geometry (Selwa et al. 2006; Gruszecki et al. 2006; Wasiljew \& Murawski 2009). This results in fundamental or second-harmonic vertical modes (depending on the location of the generated pulse), or even mixed modes of both vertical and horizontal polarisations. This observation differs from previous simulations in that the excitation of this loop comes from above rather than below the loop. It is possible that what we observe is a mixed mode oscillation but that it is dominated by the vertically polarised second or third harmonic. That the loop oscillation amplitude does not diminish completely to zero at the node point (see Fig. 2) also supports this interpretation.

\subsection{Wave speeds}

Assuming a loop length $L=466 \mathrm{Mm}$ with a nominal $10 \%$ error, the phase speed and the minimum value for the internal Alfvén speed can be estimated for different order harmonics. Table 2 gives a summary of the wave speeds calculated for both loop strands, assuming either a second or third order harmonic. The phase speed was calculated as $V_{\mathrm{ph}}=2 L / n P$, where $n$ is the order of the harmonic and an estimate of the minimum value for the internal Alfvén speed is given by $V_{\mathrm{A} 0}=V_{\mathrm{ph}} / \sqrt{ } 2$ assuming that $V_{\mathrm{ph}}$ is equal to the kink speed. For a sound speed of $500 \mathrm{~km} \mathrm{~s}^{-1}$ and an $11 \mathrm{MK}$ plasma, the maximum plasma- $\beta$ is given by $\beta=$ $\left(2 / \gamma\left(C_{\mathrm{s}}^{2} / V_{\mathrm{A} 0}^{2}\right) \approx 0.6\right.$ for the first strand $(n=3)$. Here both $V_{\mathrm{ph}}$ and $V_{\mathrm{A} 0}$ are above the sound speed for an $11 \mathrm{MK}$ plasma.

\subsection{Intensity variations}

There are various possible explanations for the intensity variations shown in Fig. 7. Firstly, intensity variations may be caused by line-of-sight effects either as a result of the oscillating loop overlapping static background structures or the change in column depth of the loop along the line of sight as it oscillates 


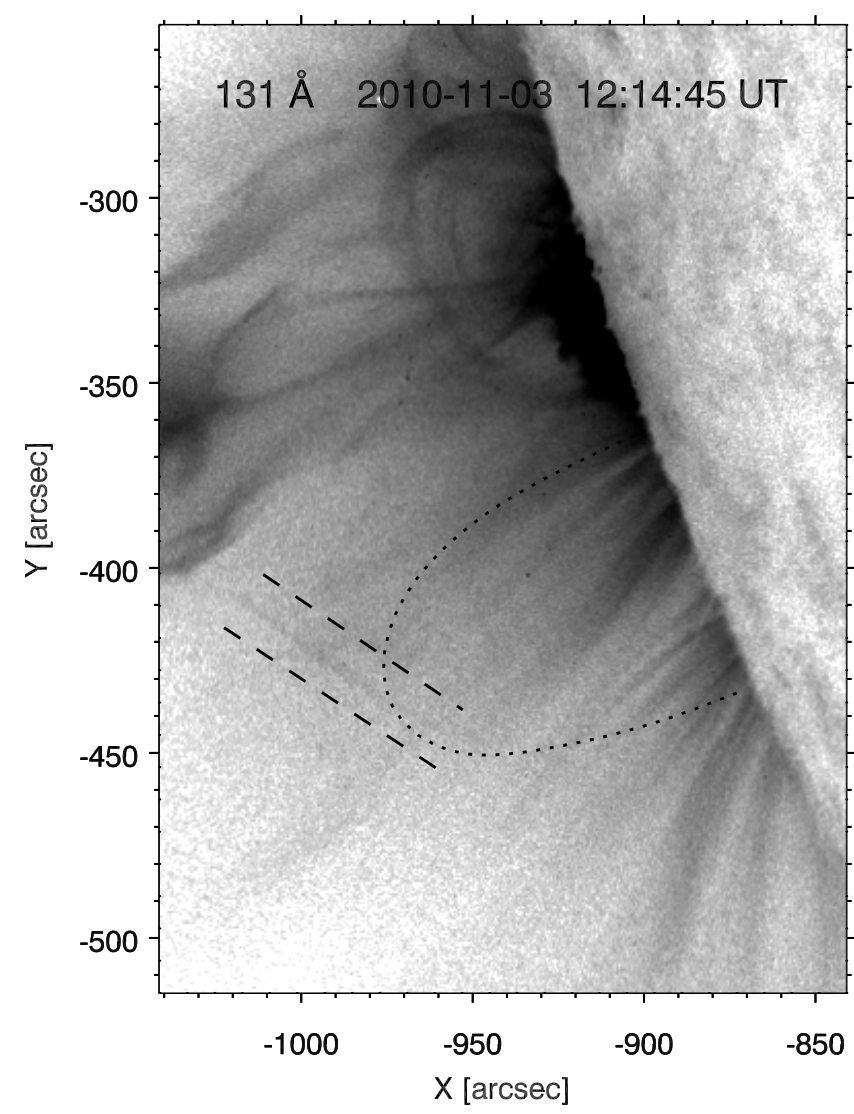

Fig. 11. An AIA image taken at 12:14:45 UT on 3 November showing a strand that appears to connect the $\mathrm{CME}$ with the region where the hot loop appears a short time later. The loop position at a time of 12:30:09 UT is indicated by the dotted line and the strand is located between the two dashed lines.

Table 2. Summary of the properties and wave speeds of the two loop strands for both a second and third order harmonic TLO.

\begin{tabular}{lccc}
\hline \hline Harmonic & & First strand & Second strand \\
\hline & $P$ & $302 \pm 14$ & $291 \pm 9$ \\
& $\tau$ & $306 \pm 43$ & $487 \pm 125$ \\
\hline$n=2$ & $V_{\mathrm{ph}}$ & $1540 \pm 170$ & $1600 \pm 170$ \\
& $V_{\mathrm{A} 0}$ & 1090 & 1130 \\
& $\beta$ & 0.3 & 0.2 \\
\hline$n=3$ & $V_{\mathrm{ph}}$ & $1030 \pm 110$ & $1070 \pm 110$ \\
& $V_{\mathrm{A} 0}$ & 730 & 760 \\
& $\beta$ & 0.6 & 0.5 \\
\hline
\end{tabular}

Notes. Parameters listed in the table: period $(P)$, damping time $(\tau)$, phase speed $\left(V_{\mathrm{ph}}\right)$, minimum internal Alfvén speed $\left(V_{\mathrm{A} 0}\right) . P$ and $\tau$ have units of $\mathrm{s}$ and all speeds have units of $\mathrm{km} \mathrm{s}^{-1}$.

back and forth (Cooper et al. 2003; Verwichte et al. 2009, 2010; White \& Verwichte 2012). This may be particularly true for the case $s=158 \mathrm{Mm}$ as this cut is taken close to the solar limb where several structures can be seen extending radially outwards. The forward modelling of the oscillating 3D loop based on a uniformly filled loop does show some variations in intensity that would support this explanation. Secondly, the mode may couple linearly with a slow mode of the same period (Terradas et al. 2011). For this, we would expect the wavelength of the slow mode to be $\lambda_{\mathrm{s}}=C_{\mathrm{s}}\left(2 L / 3 V_{\mathrm{ph}}\right)=160 \mathrm{Mm}$. We do not detect spatial intensity variations on this length-scale along the loop.
Thus, we rule out linear coupling in this case. Finally, a vertically polarised mode inherently perturbs the density as both the loop length and loop cross-section vary (Wang \& Solanki 2004; Verwichte et al. 2006a,b; Díaz 2006). Verwichte et al. (2006a) showed that the intensity perturbation for small amplitude oscillations averaged over the loop width is related to the radial displacement as $\delta I / I_{0} \approx-4 \xi / R$. For a radial displacement of 4-6 Mm, an approximate loop radius of $R=L / \pi=150 \mathrm{Mm}$, this predicts a relative intensity variation of $\delta I / I_{0}=-0.1-0.2$. The observations indeed show an intensity oscillation that is in anti-phase with the displacement with a relative amplitude between $0.1-0.3$. We therefore conclude that the detected intensity oscillations are consistent with a vertically polarised TLO.

\section{Conclusions}

We have presented the first analysis of a higher harmonic vertical transverse loop oscillation (TLO) in a hot coronal loop. The oscillation has a period of $302 \pm 14 \mathrm{~s}(291 \pm 9 \mathrm{~s})$ and a damping time of $306 \pm 43 \mathrm{~s}(487 \pm 125 \mathrm{~s})$ for the first (second) strand. Intensity variations are observed along the loop (Fig. 6) and intensity oscillations that can be fitted with a damped cosine curve are observed at two points along the loop (Fig. 7). An estimate of the 3D geometry of the loop is obtained from a comparison between STEREO-B and SDO viewpoints allowing the mode of the 5-min-period oscillation to be modelled. Our analysis can be summarised as the following key results:

1) This is the first observation of a TLO in hot coronal lines. Previous observations of TLOs had been made in the cooler bandpasses (e.g. $171 \AA$ ). This underlines the new capability of AIA/SDO of performing detailed spatial observations of the hot corona, which had been impossible with instruments such as TRACE. Coronal oscillations have been observed in hot flare lines by the SUMER spectrometer on SOHO, although in most of these cases spatial observations of the loops were unavailable. The oscillations were only observed in the Doppler shift in velocity and interpreted as slow magneto-acoustic oscillations of coronal loops. This observation clearly demonstrates that TLOs can exist in hot coronal lines. By combining high-cadence spatial observations from instruments such as AIA with spectrometer observations from HINODE/EIS (e.g. Van Doorsselaere et al. 2008), hot coronal oscillations and the distinctions between transverse and longitudinal modes may be studied in more detail.

2) The loop oscillation is vertically polarised and the detected intensity variations with relative amplitude between $0.1-0.3$ are consistent with this conclusion. The forward modelling of the oscillation also supports a vertically rather than horizontally polarised mode interpretation.

3) The loop oscillates exclusively as a harmonic overtone. A $180^{\circ}$ phase change is observed along the southern loop leg, indicating the presence of a node. The forward modelling of the different modes of oscillation suggest that the loop is oscillating either as a second or third harmonic. Since only one node is seen along the loop and the oscillations in the northern loop leg are hidden by the contrast with the background, the order of the mode cannot be confirmed. An interesting feature of this observation is that the oscillation is dominated by a higher harmonic and the fundamental mode is suppressed.

4) The excitation mechanism of this TLO in a hot loop differs from the excitation mechanism suggested for TLOs observed in cooler bandpasses. In this case, reconnection processes 
may form a post flare loop and release the loop from the top (or even push it down towards the solar surface) resulting in a vertically polarised mode. This is in contrast to the model of a blast wave hitting loops and causing transverse (often horizontally polarised) oscillations, which is often proposed as the excitation mechanism for cooler loops.

Acknowledgements. R.W. would like to acknowledge the support of a UK Science and Technology Facilities Council (STFC) Ph.D. studentship, E.V. acknowledges financial support from the STFC on the CFSA Rolling Grant and C.F. acknowledges financial support from the STFC under her Advanced Fellowship. AIA data are courtesy of SDO (NASA) and the AIA consortium. EUVI data are courtesy of LMSAL and the STEREO/SECCHI consortium.

\section{References}

Andries, J., Goossens, M., Hollweg, J. V., Arregui, I., \& Van Doorsselaere, T. 2005a, A\&A, 430, 1109

Andries, J., Arregui, I., \& Goossens, M. 2005b, ApJ, 624, L57

Aschwanden, M. J., \& Schrijver, C. J. 2011, ApJ, 736, 102

Aschwanden, M. J., Fletcher, L., Schrijver, C. J., \& Alexander, D. 1999, ApJ, 520,880

Aschwanden, M. J., de Pontieu, B., Schrijver, C. J., \& Title, A. M. 2002, Sol. Phys., 206, 99

Bain, H. M., Krucker, S., Glesener, L., \& Lin, R. P. 2012, ApJ, 750, 44

Cheng, X., Zhang, J., Liu, Y., \& Ding, M. D. 2011, ApJ, 732, L25

Cooper, F. C., Nakariakov, V. M., \& Tsiklauri, D. 2003, A\&A, 397, 765

De Moortel, I., \& Brady, C. S. 2007, ApJ, 664, 1210

Díaz, A. J. 2006, A\&A, 456, 737

Dymova, M. V., \& Ruderman, M. S. 2006, A\&A, 457, 1059

Edwin, P. M., \& Roberts, B. 1983, Sol. Phys., 88, 179

Fisher, G. H., Canfield, R. C., \& McClymont, A. N. 1985, ApJ, 289, 414

Foullon, C., Verwichte, E., Nakariakov, V. M., Nykyri, K., \& Farrugia, C. J. 2011, ApJ, 729, L8

Goossens, M., Terradas, J., Andries, J., Arregui, I., \& Ballester, J. L. 2009, A\&A, 503,213

Gruszecki, M., Murawski, K., Selwa, M., \& Ofman, L. 2006, A\&A, 460, 887

Hori, K., Ichimoto, K., \& Sakurai, T. 2007, in New Solar Physics with Solar-B Mission, eds. K. Shibata, S. Nagata, \& T. Sakurai, ASP Conf. Ser., 369, 213

Hori, K., Ichimoto, K., Sakurai, T., Sano, I., \& Nishino, Y. 2005, ApJ, 618, 1001

Howard, R. A., Moses, J. D., Vourlidas, A., et al. 2008, Space Sci. Rev., 136, 67

Kliem, B., Dammasch, I. E., Curdt, W., \& Wilhelm, K. 2002, ApJ, 568, L61

Lemen, J. R., Title, A. M., Akin, D. J., et al. 2011, Sol. Phys., 115
Lugaz, N., Downs, C., Shibata, K., et al. 2011, ApJ, 738, 127

Mariska, J. T. 2005, ApJ, 620, L67

Markwardt, C. B. 2009, in Astronomical Data Analysis Software and Systems XVIII, eds. D. A. Bohlender, D. Durand, \& P. Dowler, ASP Conf. Ser., 411, 251

McEwan, M. P., Donnelly, G. R., Díaz, A. J., \& Roberts, B. 2006, A\&A, 460, 893

McIntosh, S. W., de Pontieu, B., Carlsson, M., et al. 2011, Nature, 475, 477

Milligan, R. O., \& Dennis, B. R. 2009, ApJ, 699, 968

Milligan, R. O., Gallagher, P. T., Mathioudakis, M., \& Keenan, F. P. 2006, ApJ, 642, L169

Mrozek, T. 2011, Sol. Phys., 270, 191

Nakariakov, V. M., Ofman, L., Deluca, E. E., Roberts, B., \& Davila, J. M. 1999, Science, 285,862

Nakariakov, V. M., Tsiklauri, D., Kelly, A., Arber, T. D., \& Aschwanden, M. J. 2004, A\&A, 414, L25

Reeves, K. K., \& Golub, L. 2011, ApJ, 727, L52

Seely, J. F., \& Feldman, U. 1984, ApJ, 280, L59

Selwa, M., Solanki, S. K., Murawski, K., Wang, T. J., \& Shumlak, U. 2006, A\&A, 454, 653

Terradas, J., Andries, J., \& Verwichte, E. 2011, A\&A, 527, A132

Tothova, D., Innes, D. E., \& Stenborg, G. 2011, A\&A, 528, L12

Tsiklauri, D., Nakariakov, V. M., Arber, T. D., \& Aschwanden, M. J. 2004, A\&A, 422, 351

Van Doorsselaere, T., Nakariakov, V. M., \& Verwichte, E. 2007, A\&A, 473, 959

Van Doorsselaere, T., Nakariakov, V. M., Young, P. R., \& Verwichte, E. 2008, A\&A, 487, L17

Van Doorsselaere, T., Birtill, D. C. C., \& Evans, G. R. 2009, A\&A, 508, 1485

Verwichte, E., Nakariakov, V. M., Ofman, L., \& Deluca, E. E. 2004, Sol. Phys., 223, 77

Verwichte, E., Foullon, C., \& Nakariakov, V. M. 2006a, A\&A, 446, 1139

Verwichte, E., Foullon, C., \& Nakariakov, V. M. 2006b, A\&A, 452, 615

Verwichte, E., Aschwanden, M. J., Van Doorsselaere, T., Foullon, C., \& Nakariakov, V. M. 2009, ApJ, 698, 397

Verwichte, E., Foullon, C., \& Van Doorsselaere, T. 2010, ApJ, 717, 458

Wang, T. 2011, Space Sci. Rev., 158, 397

Wang, T. J., \& Solanki, S. K. 2004, A\&A, 421, L33

Wang, T., Solanki, S. K., Curdt, W., Innes, D. E., \& Dammasch, I. E. 2002, ApJ, 574, L101

Wang, T. J., Solanki, S. K., Innes, D. E., Curdt, W., \& Marsch, E. 2003a, A\&A, 402, L17

Wang, T. J., Solanki, S. K., Curdt, W., et al. 2003b, A\&A, 406, 1105

Wang, T., Ofman, L., Davila, J. M., \& Su, Y. 2012, ApJ, 751, L27

Wasiljew, A., \& Murawski, K. 2009, A\&A, 498, 863

White, R. S., \& Verwichte, E. 2012, A\&A, 537, A49

Witkin, A. 1983, in Proc. Int. Joint Conf. Artificial Intell., Espoo, Finland 